\title{
Mode-locked Pr3+-doped silica fiber laser with an external cavity
}

\author{
Shi, Yuan; Poulsen, Christian; Sejka, Milan; Poulsen, 0.
}

Published in:

Journal of Lightwave Technology

Link to article, DOI:

$10.1109 / 50.293964$

Publication date:

1994

Document Version

Publisher's PDF, also known as Version of record

Link back to DTU Orbit

Citation (APA):

Shi, Y., Poulsen, C., Sejka, M., \& Poulsen, O. (1994). Mode-locked Pr3+-doped silica fiber laser with an external cavity. Journal of Lightwave Technology, 12(5), 749-753. https://doi.org/10.1109/50.293964

\section{General rights}

Copyright and moral rights for the publications made accessible in the public portal are retained by the authors and/or other copyright owners and it is a condition of accessing publications that users recognise and abide by the legal requirements associated with these rights.

- Users may download and print one copy of any publication from the public portal for the purpose of private study or research.

- You may not further distribute the material or use it for any profit-making activity or commercial gain

- You may freely distribute the URL identifying the publication in the public portal

If you believe that this document breaches copyright please contact us providing details, and we will remove access to the work immediately and investigate your claim. 


\title{
Mode-locked $\mathrm{Pr}^{3+}$-Doped Silica Fiber Laser with an External Cavity
}

\author{
Yuan Shi, Christian V. Poulsen, Milan Sejka, and O. Poulsen, Member, IEEE
}

\begin{abstract}
We present a $\mathbf{P r}^{3+}$-doped silica-based fiber laser mode-locked by using a linear external cavity with a vibrating mirror. Stable laser pulses with a FWHM of less than $44 \mathrm{ps,} \mathrm{peak}$ power greater than $9 \mathrm{~W}$, and repetition rate up to $100 \mathrm{MHz}$ are obtained. The pulse width versus cavity mismatch $\Delta L$ and pump power have been investigated. With a short piece of nonlinear fiber included in the external cavity, laser pulses of 45 ps have been measured.
\end{abstract}

\section{INTRODUCTION}

$\mathbf{M}$ ODE-LOCKED lasers yield picosecond and femtosecond time resolution in a large number of applications. During the last several years, mode-locked solid-state lasers have been the subject of intensive research and improvements. Especially the mode-locked Nd:glass lasers at $1.06 \mu \mathrm{m}$ [1], [2], because of the need for front-end picosecond and femtosecond oscillators for high power $\mathrm{Nd}$ :glass amplifier chains and for simple sources of ultrashort laser pulses, have been examined. However, these mode-locked bulk solid-state laser systems suffer from thermal instability and other drawbacks.

As an attractive alternative, the rare-earth-doped fibers have been used in various mode-locked laser systems to generate ultrashort pulses. These fiber laser systems have several advantages over their bulk counterparts: They are free from thermal drifts, they show higher stability to environmental perturbations, and they could be easily integrated with fiber-optic components.

Several groups have reported mode-locking of neodymiumdoped [3]-[5] and erbium-doped [6]-[10] fiber lasers. The linear external cavity mode-locking technique [3],[11],[12] offers one simple and effective way of obtaining ultrashort pulses with high repetition rate. Further improved performance may be obtained by using a nonlinear fiber in the external cavity [13].

In this paper, we demonstrate a mode-locked $\mathrm{Pr}^{3+}$-doped fiber laser utilizing a linear and a nonlinear external cavity with a vibrating mirror. The characteristics of the laser pulses versus laser cavity parameters are investigated. The mode-locking mechanisms and dynamics are discussed.

\section{EXPERIMENTAL SETUP AND MODE-LOCKING MECHANISMS}

The schematic configuration of the mode-locked $\mathrm{Pr}^{3+}$. doped fiber laser system is shown in Fig. 1. A 108-cm-long

Manuscript received May 6, 1993; revised November 2, 1993. This work was supported by the Danish Technical Research Council (STVF)

The authors are with Mikroelektronik Centret, DTU, Bldg. 345e, DK-2800 Lyngby, Denmark.

IEEE Log Number 9215932

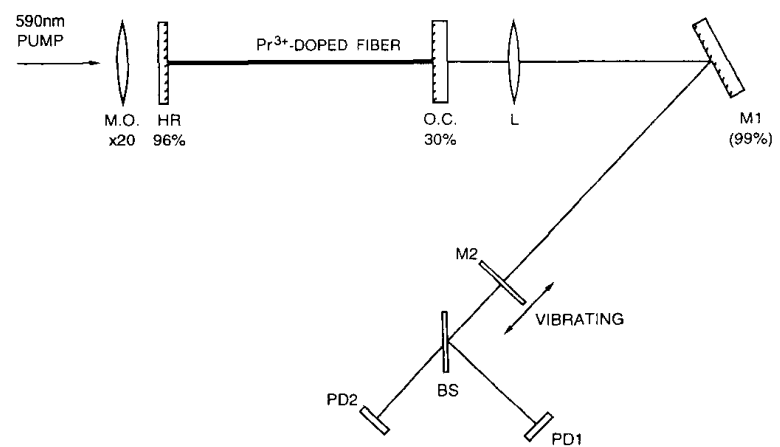

Fig. 1. Cavity configuration of the mode-locked fiber laser with an external cavity. M.O.: microscope objective. HR: high reflector. O.C.: output coupler. L: collimating lens. M1: $99 \%$ reflection mirror. M2: vibration mirror with a reflectivity of $8 \%$. BS: beam splitter. PD1 \& PD2: photodetectors.

$\mathrm{Pr}^{3+}$-doped silica-based fiber with $\mathrm{Al}$ co-dopant is used as the active laser medium. This fiber is made by a solutiondoping technique. It has a cut-off wavelength of $875 \mathrm{~nm}$, a NA of 0.18 , and a concentration of $\operatorname{Pr}^{+3}$ ions of $\approx 4.8$. $10^{18}$ ions $/ \mathrm{cm}^{3}$. The doped fiber ends are directly butted to the two laser mirrors, which form the master laser cavity. The pump of a $\mathrm{Rh} 6 \mathrm{G}$ dye laser at $590 \mathrm{~nm}$ is coupled into the doped fiber by a $\times 20$ microscope-objective through a high reflector HR (96\% reflectivity around $1048 \mathrm{~nm})$. The total coupling efficiency at the pump wavelength $590 \mathrm{~nm}$ is $\sim 60 \%$. The output from the master cavity is collimated and coupled into the external cavity by a collimating lens with a broadband antireflection coating. The external cavity is formed by the output coupler (OC), high reflection mirror M1 $(99 \%$ reflectivity around $1048 \mathrm{~nm}$ ), and mirror M2. The mirror M2, a slab of optically polished glass with $150 \mu \mathrm{m}$ thickness, yields $\sim 8 \%$ reflectivity, and is mounted on the core of a loudspeaker. The loudspeaker vibrates sinusoidally at a frequency of a few hundred $\mathrm{Hz}$. Single transverse mode emission of the laser is at $1048 \mathrm{~nm}$, corresponding to the transition of ${ }^{1} D_{2}-{ }^{3} F_{3,4}$ of the $\mathrm{Pr}^{3+}$-doped fiber.

Ultrashort pulse generation in lasers generally depends on the simultaneous presence of frequency modulation (FM) and amplitude modulation (AM) at the round trip frequency. In our mode-locked fiber laser, the external cavity, with vibrating mirror M2, gives rise to a frequency modulation, and the Kerrnonlinearity of the doped fiber yields an amplitude modulation.

The mode-locked pulse formation in the laser specifically depends on the reflecting characteristics of the external Fabry- 
Perot cavity. Considering in steady state a master cavity with many longitudinal modes, the frequency shift $\Delta \omega$, caused by the vibrating mirror, may be deduced to [14]

$$
\Delta \omega=c_{1}\left(\frac{ \pm 2 \omega_{n} V_{M}}{c}\right)
$$

where $c_{1}$ is a constant dependent on the reflections and phase delays of the two cavities, and $\pm 2 \omega_{n} V_{M} / c$ is the contribution from the Doppler shift from the linear external cavity, in which \pm represents backward and forward motion of $M_{2} ; \omega_{n}$ is the optical frequency of the n'th mode; $V_{M}$ is the translation speed of mirror $M_{2}$; and $c$ is the speed of light. The frequency shift $\Delta \omega$ is calculated to be $\sim 0.16 \mathrm{MHz}$ for a single cavity transit, when the external cavity is considered to be lossless. These frequency shifts add up for each cavity transit and will finally equal the longitudinal mode spacing of the laser $(96 \mathrm{MHz})$, resulting in mode-locking.

Amplitude modulation is required for the generation of stable pedestal-free pulses. In our case, the amplitude modulation is caused by the Kerr-nonlinearity of the $\mathrm{Pr}^{3+}$-doped fiber. The doped fiber, with its strong confinement of the optical field, gives a nonlinear optical effect. The self-phase modulation, due to the Kerr-nonlinearity in the doped fiber, gives a change in the reflection $\Delta R$ of the external cavity, which may be found as [15],[16]

$$
\Delta R=c_{2} \frac{4 \pi l}{\lambda} n_{2} I
$$

where $c_{2}$ is a constant representing the reflections and phase delays of the two cavities; $l$ is the length of the doped fiber; $\lambda$ is the lasing wavelength; $n_{2} \approx 3.2 \cdot 10^{-16} \mathrm{~cm}^{2} / \mathrm{W}$ is the nonlinear refractive index of the fiber; and $I$ is the intensity of the light in the master cavity. The fact that the change in the reflection $\Delta R$ is proportional to laser intensity implies that the reflectivity of the external cavity will increase around the center of the laser pulse. This nonlinear mechanism compresses the pulse width and gives a pulse without background noise. The relaxation time of the nonlinearity is so fast that it does not cause any limitation on the pulse width of the mode-locked laser.

With the addition of a short piece of nonlinear fiber in the external cavity, the self-phase modulation will be enhanced, and the reflection from the external cavity will be intensitydependent and will thus act as a saturable absorber mechanism. This nonlinear effect will force more laser modes to be locked and may result in an improved mode-locking performance [13].

\section{EXPERIMENTAL RESULTS AND DISCUSSIONS}

The experimental setup is shown in Fig. 1. The output of the mode-locked laser is split into two beams by the beam-splitter (BS). One beam is sent to a monitoring photodetector (PD1) with a bandwidth of $500 \mathrm{MHz}$ for the optimization of the laser with respect to the pulse intensity. Another beam is used for analyzing the laser pulse shape by a fast photodetector (PD2). With a $30 \%$ OC, the fiber laser has a cw lasing threshold of $4 \mathrm{~mW}$ (absorbed pump power), and a slope efficiency of $18 \%$, measured immediately after mirror M2. The threshold of mode-locked operation is $50 \mathrm{~mW}$, much higher than the

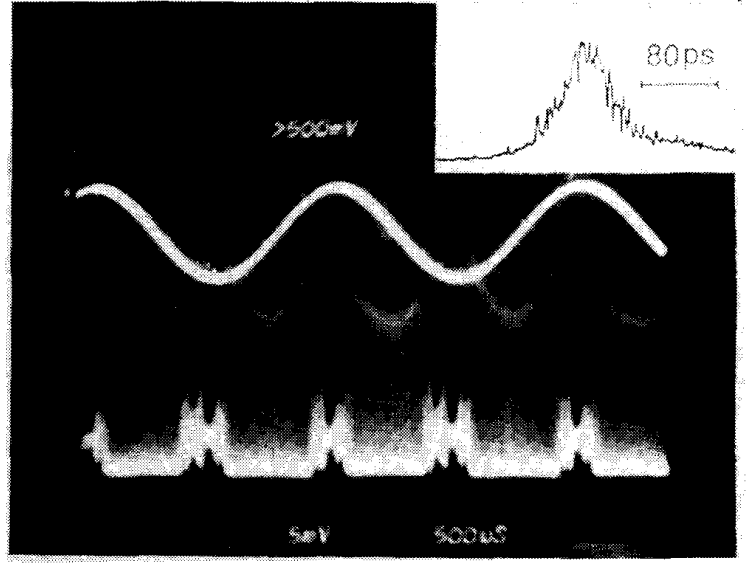

Fig. 2. The mode-locked performance of the fiber laser is shown. The lower trace shows the mode-locked pulse trains. The sinusoidal drive signal to mirror M2 is shown in the upper trace. The time scale is $500 \mu \mathrm{s} / \mathrm{div}$. The insert shows a single mode-locked pulse with a pulse width of $50 \mathrm{ps}$.

threshold of cw-lasing, because the mode-locking operation can only be achieved with many modes excited and with a pronounced Kerr nonlinearity. Pulse width and stability of the mode-locked pulse train has been studied as function of the modulation frequency of mirror M2. Mode-locked operation is observed for modulation frequencies of the mirror in the range of $100-450 \mathrm{~Hz}$. Fig. 2 shows a pulse train obtained at a driving frequency of $300 \mathrm{~Hz}$, where optimum conditions as to pulse width and stability are found. The periodicity of the modelocked pulse train corresponds to one sweep of the mirror, with mode-locking rapidly building up and decaying around the turning points of the mirror (where the Doppler shift is zero). The difference in amplitude and duration of the pulsetrain envelopes are due to different off-axis displacements of the loudspeaker during its forward and backward movement. The mode-locked laser pulses have a repetition rate of $96 \mathrm{MHz}$, corresponding to the free spectral range of the master laser cavity.

The pulse width measurement system of the mode-locked laser consists of a $40 \mathrm{GHz}$ optical front-end photodetector and a fast digital oscilloscope with a bandwith of $34 \mathrm{GHz}$. This system has a measured FWHM response time of 14 ps for the photodetector and oscilloscope using an additive-pulse mode-locked femtosecond laser as the fast source. The pulse width (FWHM) of the mode-locked fiber laser is obtained by deconvoluting the measured pulse width with the prompt of the detection system. Due to reflections from the measurement systems, spurious optical feedback must be below $\approx-30$ $\mathrm{dB}$ to avoid deteriorating mode-locked operation. Thus the measurement system is misaligned to avoid this problem. Several parameters control the quality of the mode-locked pulses, the most important being the cavity mismatch $\Delta L$ (the difference in optical length between the master cavity and the external cavity), the output coupling, and the feedback from the external cavity. Fig. 3 shows typical output pulses as function of cavity mismatch $\Delta L$; and Fig. 4 shows the full dependence of the pulse width on cavity mismatch for two values of the output couplers (OC): $30 \%$ and $44 \%$ respectively. 


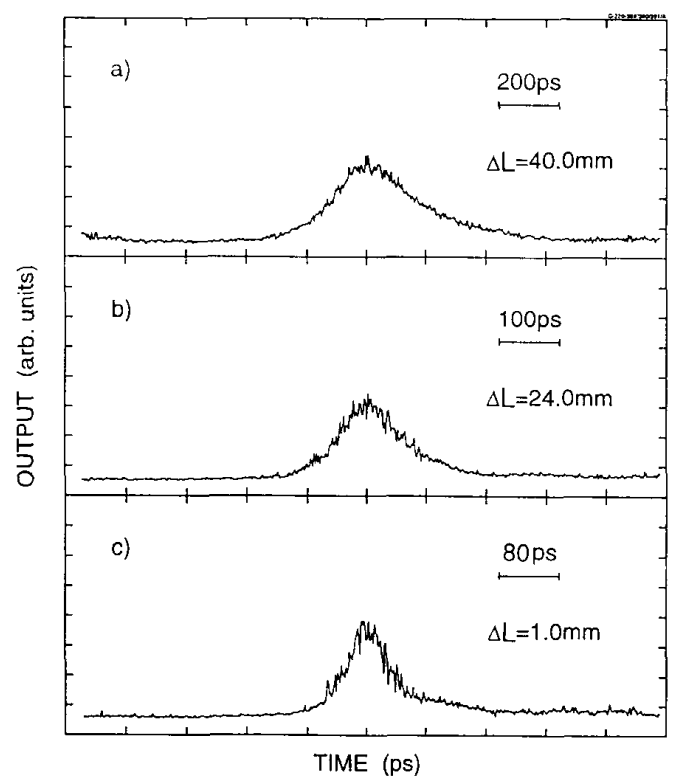

Fig. 3. Three typical output laser pulses as function of cavity mismatch $\Delta L$. The output coupling is OC $=30 \%$.

The pulses are all measured with a fiber length of $108 \mathrm{~cm}$, a pump power of $216 \mathrm{~mW}$, and a vibration frequency of $300 \mathrm{~Hz}$ of the mirror M2. Mode-locking ceases for cavity detuning below $0.4 \mathrm{~mm}$. This is in agreement with other investigations [3],[17] employing different cavity configurations and laser media. The reason for the disappearance of mode-locking is attributed to the extension of the stationary field from the master laser cavity to the external cavity due to mode matching of the two cavities. The resulting interference prevents the locking of the modes even in the presence of a frequency chirp due to the vibrating mirror M2. For cavity detuning larger than $0.4 \mathrm{~mm}$, the pulse width of the mode-locked laser becomes larger with increasing cavity detuning. This is due to the fact, that for the larger cavity detuning, it takes a longer time to find a balanced stable mode-locking point. The $44 \%$ $\mathrm{OC}$ results in wider pulses and a faster broadening of the pulse width as a function of the cavity mismatch, as compared to $30 \%$ OC. Positive and negative cavity mismatch, which result in a different broadening rate, has been investigated in [18]. Satellite peaks in the laser pulse, caused by the interference between the field inside the master cavity and the field reflected back from the external cavity [3], become pronounced with $44 \%$ OC.

The 30\% OC yielded pulse width less than $44 \mathrm{ps}$ and an average power of $40 \mathrm{~mW}$. Peak power and pulse energy are found to be $9 \mathrm{~W}$ and $0.4 \mathrm{~nJ}$ respectively. The $44 \%$ OC resulted in a pulse width of $56 \mathrm{ps}$. To further study the effect of the output coupling, a $15 \%$ OC is used, which gives a pulse width of 90 ps. Thus, $15 \%$ OC does not result in a better modelocking, despite of the expectation of having a larger number of longitudinal modes and an enhanced nonlinearity. A lower coupling efficiency between the master and the external cavity causes this deterioration of the mode-locked performance.
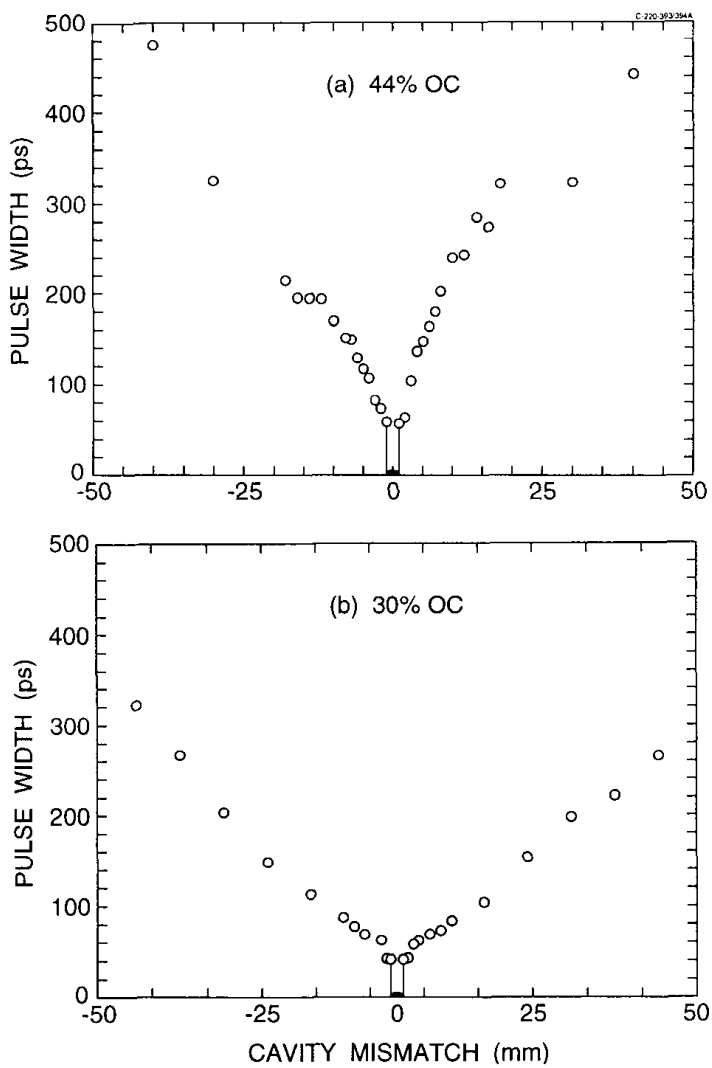

Fig. 4. The pulse width versus cavity mismatch. (a) $44 \%$ output coupler (b) $30 \%$ output coupler. Pulses as short as $\mathbf{4 4}$ ps are observed.

The pulse width and the length of the pulse trains are found to be sensitive to the amplitude and frequency of the vibrating mirror M2. With a higher amplitude, where mirror M2 has a higher translation speed, a slightly longer pulsetrain is expected from the mode-locked laser. However, our experimental results are in contradiction to this analysis. The reason is related to the stability of the vibrating mirror. We observe persistent relaxation oscillation with a several-kHz repetition rate, when the vibrating mirror $\mathrm{M} 2$ is not carefully adjusted. This is due to a non-uniform transverse vibration of the loudspeaker.

The output pulse width as a function of absorbed pump power is investigated for a fiber length of $94 \mathrm{~cm}$, a vibration frequency of $350 \mathrm{~Hz}$ of the mirror M2, and a cavity detuning of $3.0 \mathrm{~mm}$. The results are shown in Fig. 5. The pulse width is found to be insensitive to pump power. This is due to two competing factors affecting the mode-locked pulse width. A higher pump power tends to stimulate more oscillating modes, and the nonlinear effect in the doped fiber becomes stronger, giving a larger amplitude modulation. These two effects force the laser pulses to become narrower. On the other hand, higher pump power also narrows the stimulated emission spectrum, resulting in a broadening of the laser pulse width.

To enhance the nonlinear effect we have included a 28 $\mathrm{cm}$-long nonlinear fiber in the external cavity. The feedback parameters of the external cavity have been changed in order 


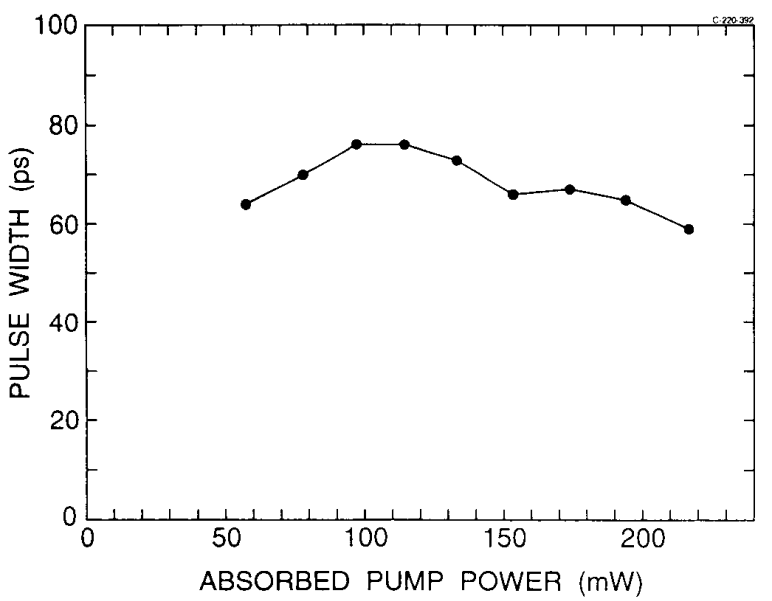

Fig. 5. The output laser pulse width versus absorbed pump power for $30 \%$ $O C$ with a doped fiber length of $94 \mathrm{~cm}$, a vibration frequency of $350 \mathrm{~Hz}$ of mirror $\mathrm{M} 2$, and a cavity detuning of $3.0 \mathrm{~mm}$.

to yield $\sim 10 \%$ feedback. By careful adjustment of the system to reduce the spurious inter-reflection, the mode-locked laser with a FWHM of 45 ps has been obtained. The performance of the mode-locked laser has not been improved, compared to the laser with the linear cavity. This is because of lower stability and the spurious internal reflections of the laser system.

\section{CONCLUSION}

We have demonstrated the mode-locked $\mathrm{Pr}^{3+}$-doped silica fiber laser by employing a linear external cavity with a vibrating mirror. Stable trains of mode-locked pulses with pulse width less than 44 ps, peak powers larger than $9 \mathrm{~W}$, and pulse energies larger than $0.4 \mathrm{~nJ}$, have been achieved. The mode-locked pulses exhibited a high stability to environmental perturbations. With a nonlinear external cavity, laser pulses of $45 \mathrm{ps}$ have been obtained. Thus the $\mathrm{Pr}^{3+}$-doped silicabased mode-locked fiber laser is found to be attractive as an ultrashort pulse generator in many applications. Furthermore, with the decrease of fiber length in the laser cavity, the modelocked fiber laser system can offer ultrashort laser pulses at a very high repetition rate.

\section{ACKNOWLEDGMENT}

The authors are grateful to $\mathrm{C}$. Larsen and B. Palsdottir, LYCOM A/S, for providing the $\mathrm{Pr}^{3+}$-doped fiber.

\section{REFERENCES}

[1] F. Krausz, T. Brabec, E. Wintner, and A. J. Schmidt, "Mode locking of a continuous wave Nd:glass pumped by a multistripe diode laser," Appl. Phys. Lett., vol. 55, pp. 2386-2388, Dec. 1989.

[2] C. Spielmann, F. Krausz, T. Brabec, E. Wintner, and A. J. Schmidt, "Femtosecond passive mode locking of a solid-state laser by a dispersive balanced nonlinear interferometer," Appl. Phys. Lett., vol. 58, pp. 2470-2472, June 1991

[3] G. Sargsjan, V. Stamm, C. Unger, and W. Zschocke, "Characteristics of a neodymium-doped fiber laser mode-locked with a linear external cavity," Opt. Commun., vol. 86, pp. 480-486, Dec. 1991

[4] M. Hofer, M. E. Fermann, F. Haberl, and J. E. Townsend, "Active mode-locking of a neodymium-doped fiber laser using intracavity pulse compression," Opt. Lett., vol. 15, pp. 1467-1469, Dec. 1990.
[5] M. E. Fermann, M. Hofer, F. Haberl, A. J. Schmidt, and L. Turi, "Additive-pulse-compression mode-locking of a neodymium-doped fiber laser," Opt. Lett., vol. 16, pp. 244-246, Feb. 1991.

[6] A. Takada and H. Miyazawa, "Erbium fiber soliton laser," Electron. Lett., vol. 26, pp. 216-217, Feb. 1990.

[7] R. P. Davey, N. Langford, and A. 1. Ferguson, "Subpicosecond pulse generation from erbium doped fiber laser," Electron. Lett., vol. 27, pp. 726-728, Apr. 1991.

[8] R. P. Davey, R. P. E. Fleming, K. Smith, R. Kashyap, and J. R. Armitage, "Mode-locked erbium fiber laser with wavelength selection by means of fiber Bragg grating reflector," Electron. Lett., vol. 27, pp. 2087-2088, month,1991.

[9] I. N. Duling "Subpicosecond all-fiber erbium laser," Electron. Lett., vol. 27, pp. 542-544, month, 1991

[10] S. J. Frisken, C. A. Telford, R. A. Betts, and P. S. Atherton, "Passively mode-locked erbium-doped fiber laser with nonlinear fiber mirror," Electron. Lett., vol. 27, pp. 887-889, May 1991.

[11] P. G. J. Wigley, P. M. W. French, and J. R. Taylor, "Mode locking of a continuous wave neodymium doped fiber laser with a linear external cavity," Electron. Lett., vol. 26, pp. 1238-1240, Aug. 1990.

[12] C. Chaptain Cutler, "Why does linear phase shift cause mode locking," IEEE J. of Quant. Electron., vol. 28, pp. 282-288, Jan. 1992.

[13] K. J. Blow and D. Wood, "Mode-locked lasers with nonlinear external cavity," J. Opt. Soc. Amer. (B), vol. 5, pp. 629-632, Mar. 1988.

[14] A. Siegman, Lasers. Mill Valle, CA: Univ. Science Books, 1986, p. 526

[15] M. E. Ferman, F. Haberl, M. Hofer, and H. Hochreiter, "Nonlinear amplifying loop mirror," Opt. Lett., vol. 15, pp. 752-754, Feb. 1990.

[16] F. Krausz, C. Spielmann, T. Brabec, E. Wintner, and A. J. Schmidt, "Self-starting additive-pulse mode locking of a Nd:glass laser," Opt. Lett., vol. 15, pp. 1082-1084, Oct. 1990.

[17] P. M. J. French, S. M. J. Kelly, and J. R. Taylor, "Mode locking of a continuous-wave titanium-doped sapphire laser using a linear external cavity," Opt. Lett., vol. 15, pp. 378-380, Apr. 1990.

[18] M. J. Kelly, "Mode-locking dynamics of a laser coupled to an empty external cavity," Opt. Commun., vol. 70, pp. 495-501, Apr. 1989.

Yuan Shi was born in Shaanxi Provino, China, in 1962. He received the B Sc. degree from the Physics Department of Sichuan University in July, 1984 and the M. Sc. degree from Xi' an Institute of Optics and Precision Mechanics of Academia Sinica in July, 1987. He is now working towards the Ph. D. degree in photonics at the Mikroelektronik Centret of the Technical University of Denmark. His research interests are new fiber lasers and amplifiers, and crystal lasers.

From 1987 to 1990, he was a Research Associate at the Xi'an Institute. working with liquid crystal spatial light modulators and optoelectric hybrid image recognition systems.

Christian V. Poulsen was born in Copenhagen, Denmark, on March 17, 1968 He received the $M$. Sc. degree in electrical engineering from the Electromagnetics Institute, Technical University of Denmark, in 1992. Working toward his Ph. D. degree at the Mikroelektronik Centret at the Techical University of Denmark, he is presently investigating active waveguides. 
Milan Sejka was born in Banska Bystrica, Slovakia, on July 3, 1966. He received the $M$. Sc. degree in material science from the Slovak Technical University, Bratislava, in 1989. From 1989 to 1991 , he was a research scholar at the Department of Material Science, Slovak Technical University, working on parametrisation of optical fibers and cables. At present, he is working toward the Ph. D. degree at the Mikroelektronik Centret at the Technical University of Denmark.

From 1991 to 1992, he was with the NKT Research Center, Denmark, working on the development of erbium-doped fiber lasers and investigating fiber optic components. His current research interests include development and characterization of novel rare-earth-doped fiber laser sources and nonlinear fiber optics.

Mr. Sejka is a member of the IEEE Lasers and Electro-Optics Society and the Optical Society of America.
Ove Poulsen (M'92) received the Ph. D. degree from the University of Aarhus in 1976, followed by three years of post-doctoral work at JLA and the Argonne National Laboratory. He received the Dr. Scient. degree in 1984.

Dr. Poulsen became a Research Professor of experimental atomic and lase physics in 1986, and a Full Professor at Aarhus University, and managing director of the Mikroelektronik Centret at the Technical University of Denmark in 1991. At present, he is working with laser physics and technology, and laser cooling of atomic ions.

Dr. Poulsen is a member of IEEE. 